\title{
Does lacking information about your affordances impact your perception of others' affordances? A test of the embodied simulation hypothesis
}

\author{
Keith S. Jones ${ }^{1} \cdot$ Benjamin P. Widlus ${ }^{1} \cdot$ Nicholas A. Garcia ${ }^{1}$ \\ Published online: 26 February 2020 \\ (C) The Psychonomic Society, Inc. 2020
}

\begin{abstract}
It has been argued that observers perceive actors' affordances via embodied simulation, that is, first perceiving their own affordance, which serves as a model for the actor's affordance, and then adjusting that model to account for differences between themselves and the actor. If so, then preventing observers from picking up information about their own affordances should cause several effects. Specifically, observers should make more errors about the actor's affordance compared to when the observer is free to pick up information about their own affordance. In addition, judgments about the actor's affordance should align better with the observer's affordance than with the actor's affordance, and increase in error as differences between the observer's and actor's affordances increase. The present study tested those predictions. To do so, observers (participants) made judgments about the farthest distance that an actor (a confederate) could reach. The observer's arms were either free to move or were immobilized by having the participant hold them behind their back. The present results did not support the predictions. The present research introduces a novel means for evaluating the Embodied Simulation Hypothesis, provides initial tests of related predictions, and corroborates prior research. In addition, it motivates important questions about embodied simulation and affordance perception.
\end{abstract}

Keywords Visual perception $\cdot$ Embodied cognition

\section{Introduction}

An affordance is a relational property of an actor-environment system that determines whether an action is possible (Gibson, 1979/1986; Stoffregen, 2003). For example, the relation (affordance) between the distance a person can extend their arm in front of them and the distance between that person and an object determines whether that person can reach that object.

Gibson (1979/1986) argued people perceive affordances, including those between another person and their environment that determine whether that other person can perform certain actions. Subsequent research demonstrated observers can perceive whether actors can sit on something (Stoffregen, Gorday, Sheng, \& Flynn, 1999), step across a gap (Mark, 2007), climb something in a bipedal fashion (Mark, 2007),

Keith S. Jones

keith.s.jones@ttu.edu

1 Department of Psychological Sciences, Texas Tech University, 2810 18th St., Lubbock, TX 79409-2051, USA and reach something (Fischer, 2003; Ramenzoni, Riley, Davis, Shockley, \& Armstrong, 2008a; Ramenzoni, Riley, Shockley, \& Davis, 2008b, 2008c).

Some argue observers perceive actors' affordances via embodied simulation (Gallese \& Sinigaglia, 2011). From this perspective, an observer perceives an actor's affordance by first perceiving their own affordance, which serves as a model for the actor's affordance, and then adjusting that model to account for observer-actor differences (Gallese \& Sinigaglia, 2011). For example, to perceive whether an actor can reach something, an observer would perceive their own reach, and then adjust that model to account for observer-actor differences.

Affordance perception via embodied simulation has empirical support. For example, manipulating observers' affordances influenced their judgments about actors' affordances (Ramenzoni et al., 2008b). Observers estimated the maximum height to which actors could jump to reach an object, once without weights and again with weights attached to their ankles that substantially reduced observers' jumping capabilities. Observers' estimates were lower when observers wore ankle weights compared to when observers did not, 
which suggests observers used their own affordances to model the other person's affordance.

In the present experiment, we sought converging evidence for affordance perception via embodied simulation. We did so, not by manipulating observers' affordances (Ramenzoni et al., 2008b), but by preventing observers from generating certain kinds of information about their own affordances.

Gibson (1979/1986) argued people pick up information about affordances. Consistent with that argument, preventing people from picking up information about affordances caused them to make more errors compared to when they were free to pick up information (Jones \& Widlus, in press; Mantel, Stoffregen, Campbell, \& Bardy, 2015; Mark, Balliett, Craver, Douglas, \& Fox, 1990). For example, Jones and Widlus (in press) demonstrated preventing people from moving their arms, which reveals arm length information (Turvey \& Carello, 2011), led people to make more erroneous judgments about whether they could reach something compared to when they were free to move their arms. This was true even though they had substantial reaching experience, which suggests people pick up information about their affordances each time they perform a given action (Mark et al., 1990).

Such results provide a novel way to test for affordance perception via embodied simulation. If an observer uses their own affordance as a model for an actor's affordance, then preventing the observer from picking up information about their own affordance should cause them to make more errors about the actor's affordance compared to when the observer is free to pick up information about their own affordance.

The present experiment tested that possibility. Observers (participants) made judgments about the farthest distance that an actor (a confederate) could reach. The participant's arms were either free to move (Unrestricted Condition) or immobilized by having the participant hold them behind their back (Restricted Condition). If an observer uses their own affordance as a model for an actor's affordance, then preventing an observer from moving their arms should cause them to make significantly more errors about an actor's reach compared to when the observer is free to move their arms.

The present experiment also tested two other predictions. If an observer uses their own reach as a model for an actor's reach, then their judgments about the actor's reach should (1) align significantly better with the observer's reach than with the actor's reach, and (2) significantly increase in error as differences between the observer's and actor's reaches increase. The former reflects that an observer should be better at determining their own reach than adjusting knowledge about their reach to account for differences between them and the actor. The latter reflects that observers who perceive others' affordances via embodied simulation should be less capable of judging others' affordances when they differ from the observers' affordances (Fischer, 2003; Ramenzoni et al., 2008a).
To our knowledge, advocates of the Embodied Simulation Hypothesis have not proposed these predictions. However, they are logically consistent with standard accounts of the hypothesis (Gallese \& Sinigaglia, 2011).

\section{Method}

\section{Participants}

Thirty-four students participated (20 females; 31 right-handed). Their ages ranged from $18-22$ years $(M=19.21, S D=$ 1.09), their shoulder heights, measured from the acromion process on their dominant shoulder, ranged from 1,284.7$1,602.3 \mathrm{~mm}(M=1402.06, S D=84.68)$, and their actual maximum reaches ranged from 924.6-1,183.6 $\mathrm{mm}(M=$ $1060.11, S D=67.03)$. All received course credit.

\section{Confederate}

The confederate was a 22-year-old female who was free from motor impairments and right-handed. Her dominant shoulder height was $1,327 \mathrm{~mm}$, and her actual maximum reach was $964 \mathrm{~mm}$.

\section{Experimental design}

A within-subjects design was employed. The independent variable was Observer Arm Movement. Participants made judgments about the confederate's reach while participants' arms were free to move (Unrestricted Condition) or immobilized by grasping their non-dominant wrist with their dominant hand behind their backs (Restricted Condition). Condition order was counterbalanced. The confederate's arm movements were unrestricted. The dependent variable was the distance between the confederate's clavicle and the to-be-reached object once participants were satisfied the confederate was positioned as far away from the hanging object as she could possibly be while still being able to grasp it with her dominant hand, with both feet touching the ground and without falling over, or hitting the object so that it swung back to her. Those distances served as the basis for several different measures, which are detailed in the Results section.

\section{Apparatus}

Testing occurred in a $4.57 \mathrm{~m} \times 4.27 \mathrm{~m}$ room; black curtains covered its walls. A cardboard cylinder (height $=9.53 \mathrm{~cm}$, diameter $=3.18 \mathrm{~cm}$ ) with a retroreflective marker (diameter $=2.54 \mathrm{~cm}$ ) protruding from its top was suspended vertically (height $=1327 \mathrm{~mm}$; the height of the confederate's dominant shoulder) by a thin white string at the room's rear corner, perpendicular to and $1.83 \mathrm{~m}$ away from each of the connecting 
walls. That cylinder was the to-be-reached object for judgment trials, and when determining the participants' and confederate's actual maximum reach distances.

The participant's and confederate's movements were recorded using an eight-camera optical motion capture system, which sampled at $100 \mathrm{~Hz}$ (Software: Nexus 1.8; Cameras: MX T10, Vicon Motion Systems, Oxford, UK). The participant and confederate each wore a jacket, to which retroreflective markers ( $1.70 \mathrm{~cm}$ in diameter) were placed in accordance with Vicon's guidebook (Vicon, ND). The confederate also wore a cap with markers on its brim and apex. The motion capture system was used to position the participant and confederate before each trial, and record perceived and actual maximum reach distances.

\section{Procedure}

This research complied with the American Psychological Association Code of Ethics. Texas Tech University's Institutional Review Board approved the research protocol.

Testing lasted approximately $1.5 \mathrm{~h}$. Before participants arrived, the confederate donned her motion capture jacket and cap, and removed her shoes.

Before entering the testing room, the participant provided informed consent, donned their motion capture jacket, and removed their shoes. The participant entered the testing room; the to-be-reached object was not visible. The experimenter used the motion capture system to record the participant's dominant shoulder height. The participant then left the testing room, at which time the experimenter hung the to-be-reached object. The participant then began their first condition. Condition order was counterbalanced.

Unrestricted condition Participants were told they would make judgments about the confederate's maximum reach by verbally directing the confederate to move toward or away from the to-be-reached object until the confederate was positioned at what the participant perceived to be the confederate's maximum reach distance. Participants were told to stand normally with their arms by their sides; they were not told to move their own arms during trials. The experimenter (not the confederate) then demonstrated a maximum reach, and explained that no one would perform a maximum reach during the experiment's judgment phase.

Participants were then told they would make judgments from different locations, the experimenter would reposition the participant and confederate after each trial, and participants would be positioned first. Participants were also told to keep their eyes closed while being positioned, and to perform the task as quickly as possible and without overthinking it (Heft, 1993).

Participants then completed a practice trial; participants were not told this was practice. Data from practice trials were not analyzed. The experimenter positioned the participant at a location that was $609.6 \mathrm{~mm}(2 \mathrm{ft})$ from, to the left of, and perpendicular to the midpoint between where the confederate would stand and the to-be-reached object (Fig. 1). The experimenter then positioned the confederate. This location was used for every practice trial. Next, the participant was instructed to open their eyes, pivot towards the midpoint between the confederate and the object, and begin directing the confederate. The participant directed the confederate until the participant was satisfied. The confederate's arms swung freely by her sides and moved when she did. Participants' arms also swung freely by their sides. The distance between the confederate and the to-be-reached object was then recorded. Afterward, the experimenter corrected misunderstandings.

Participants then completed nine experimental trials. For each, the confederate was placed at a unique starting location, which varied to reduce the likelihood the participant would memorize the number of steps needed to direct the confederate to her maximum reach distance. To determine the nine confederate starting locations, different starting distances from each of the following ranges: $2.54-60.96 \mathrm{~cm}, 63.50-121.92$ $\mathrm{cm}, 124.46-182.88 \mathrm{~cm}$, and different starting angles, from each of the following ranges: $0-29^{\circ}, 30-59^{\circ}, 60-89^{\circ}$, were randomly selected and paired without replacement. In total, three random distances from each of the three distance ranges (nine total distances) were randomly selected and paired with three random angles from each of the three angle ranges (nine total angles) to make nine unique angle/distance combinations. Starting distance and angle combinations were balanced to avoid the possibility that participants would be positioned at, for example, only close distances or extreme angles. All experimental trials were identical to the practice trial, other than the confederate's starting locations and sometimes the positioning of the participant relative to the confederate. The participant was positioned to the confederate's right when positioning them to the confederate's left would place them too close to the motion camera equipment. After completing all trials, participants exited the testing room.

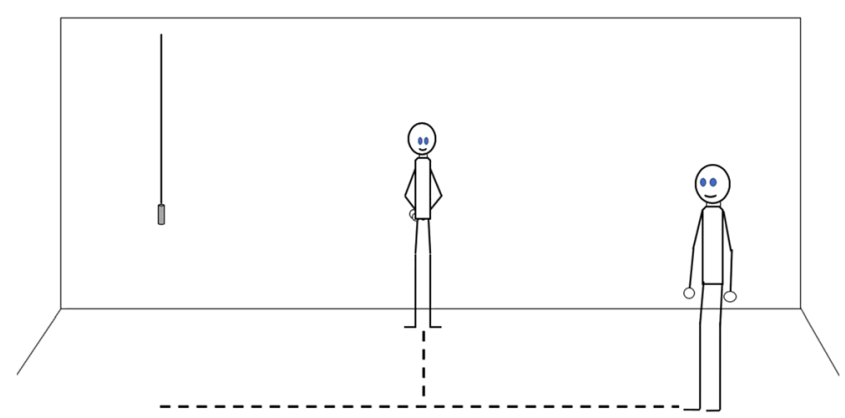

Fig. 1 A depiction of the relative position of an observer and the confederate at the beginning of a trial in the Restricted Condition. The dotted lines are provided here to highlight that the observer was positioned at the mid-point between the object and actor; they were not present during testing 
Restricted condition Data collection in the Restricted Condition was identical to that in the Unrestricted Condition, except participants kept their arms behind their backs until the condition was complete. After completing all trials, participants left the testing room.

Maximum reach measurement After completing both conditions, the experimenter adjusted the height of the object to the participant's dominant shoulder height. The participant then re-entered the testing room and the experimenter re-explained the maximum reach definition. The participant positioned themselves at what they believed to be their maximum reach distance and attempted to reach the object. After successes, the experimenter instructed the participant to move away from the object; after failures, the experimenter instructed the participant to move closer to it. This process was repeated until the experimenter recorded a successful maximum reach that was within $12.7 \mathrm{~mm}(.5 \mathrm{in}$.) or less from the longest failed reach attempt. The distance between the participant and the to-bereached object was recorded, and was considered the participant's maximum reach distance.

\section{Results}

\section{Outlier analyses}

Judgments were converted into four measures. Each was subjected to an outlier analysis, which followed Stevens (2009) recommendations. Two outliers were identified and winsorized. Winsorized and non-winsorized results were comparable.

\section{Analytic approach}

Three t-tests were conducted. To control inflation of Type I error, those tests were designated a family, and family-wise alpha was maintained at .05 via a Bonferroni correction (Kirk, 1995). Each t-test was evaluated against a per comparison alpha of $.05 / 3=.017$. One-tailed tests were employed because all predictions were directional (Kirk, 1995). All tests were conducted using IBM SPSS 25.

\section{Did restricting arm movements increase judgment error?}

Prediction If observers' judgments of the confederate's reaching capabilities were based on observers' capabilities, then arm restriction should significantly increase judgment error.

Dependent variable Absolute Judgment Error ${ }_{\text {Confederate was }}$ the percentage of absolute error between the participant's judgment of the confederate's maximum reach and the confederate's actual maximum reach (| (judged maximum distance confederate could reach / actual maximum distance confederate could reach) $-1 \mid) * 100$. Removing the sign prevents over- and underestimations from cancelling one another when data were averaged, which would incorrectly suggest participants were accurate. Within each experimental condition, Absolute Judgment Error ${ }_{\text {Confederate was averaged across trials }}$ to produce a mean Absolute Judgment Error Confederate $_{\text {for each }}$ participant.

Statistical test A one-tailed paired-samples t-test evaluated

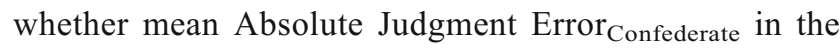
Unrestricted Condition ( $M=18.31 \%, S D=12.13 \%)$ was less than Absolute Judgment Error Confederate $_{\text {in the Restricted }}$ Condition $(M=16.32 \%, S D=11.01 \%)$. That $\mathrm{t}$-test was not statistically significant, $t(33)=1.694, p=.950$, which suggests arm restriction did not significantly increase judgment error (Fig. 2). The reported p-value was computed as 1-.05 (the one-tailed probability ordinarily reported for \pm 1.694 ) because the observed mean difference was positive whereas the one-tailed t-test could only detect negative mean differences (Cohen, 2013). It is unlikely that this outcome reflects low statistical power because the observed mean difference was in the opposite direction from what was predicted.

\section{Did judgments align better with observers' or the confederate's capabilities?}

Prediction If observers' judgments of the confederate's reaching capabilities were based on observers' capabilities, observers' judgments should align with observers' capabilities better than with the confederate's. If so, judgment error computed as a function of the observers' capabilities, Absolute Judgment Error ${ }_{\text {Participant, }}$, should be significantly less than Absolute Judgment Error ${ }_{\text {Confederate. }}$.

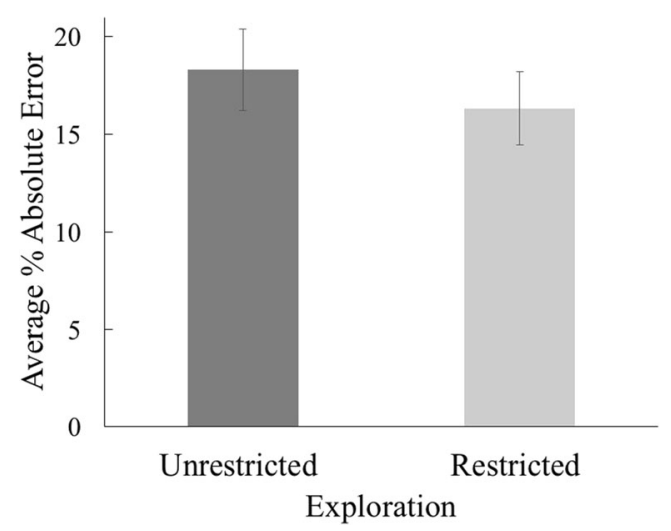

Fig. 2 Comparison of Absolute Judgment Error $_{\text {Confederate }}$ in the Unrestricted and Restricted Conditions. Error bars represent \pm 1 standard error units 
Dependent variable Absolute Judgment Error ${ }_{\text {Participant }}$ was the percentage of absolute error between the participant's judgment of the confederate's maximum reach and the participant's actual maximum reach (| (judged maximum distance confederate could reach / actual maximum distance participant could reach) $-1 \mid) * 100$. Within each experimental condition,

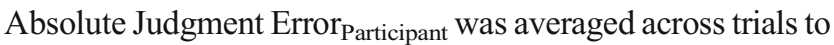

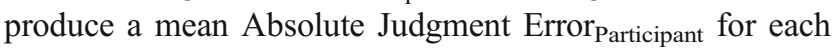
participant. Those values were then averaged across conditions because the preceding analysis failed to reveal a statistically significant difference between the Unrestricted and Restricted Conditions.

Statistical test A one-tailed single-sample t-test evaluated whether mean Absolute Judgment Error $_{\text {Participant }}(M=$ $21.47 \%$, $S D=10.58 \%$ ) was less than the grand mean of

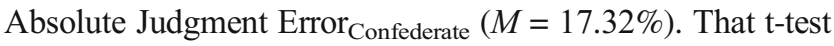
was not statistically significant, $t(33)=2.288, p=.985$, which suggests observers' judgments did not align with observers' capabilities better than with the confederate's. The reported $\mathrm{p}$ value was computed as $1-.014$ (the one-tailed probability ordinarily reported for \pm 2.288 ) because the observed mean difference was positive whereas the one-tailed t-test could only detect negative mean differences (Cohen, 2013). It is unlikely that this outcome reflects low statistical power because the observed mean difference was in the opposite direction from what was predicted, and the test would have been statistically significant had a positive mean difference been predicted.

\section{Did judgment error correlate with the degree of dissimilarity between observers' and the confederate's capabilities?}

Prediction If observers' judgments of the confederate's reach were based on observers' reaches, then as observers' reaches diverge from the confederate's, observers' reaches should increasingly become a poor means of estimating the confederate's reach, which should result in a corresponding increase in judgment error. In that case, judgment error computed as a function of the confederate's reach, Absolute Judgment Error $_{\text {Confederate, should significantly and positively correlate }}$ with a measure of how dissimilar the observers' and confederate's reaches were, Participant-Confederate Reach Dissimilarity.

Dependent variable Participant-Confederate Reach Dissimilarity was the absolute ratio between the maximum distance participants could reach and the maximum distance the confederate could reach (||actual maximum distance participant could reach / actual maximum distance confederate could reach) $-1 \mid) * 100$. Absolute Judgment Error Confederate, $_{\text {, }}$ collapsed across the Unrestricted and Restricted Conditions, was also employed.
Statistical test A one-tailed t-test evaluated whether a statistically significant positive correlation between Absolute Judgment Error ${ }_{\text {Confederate }}$ and Participant-Confederate Reach Dissimilarity occurred. That t-test was not statistically significant, $t(32)=-2.469, p=.99$, which suggests

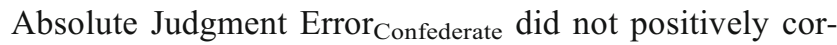
relate with Participant-Confederate Reach Dissimilarity (Fig. 3). The reported p-value was computed as 1-.010 (the one-tailed probability ordinarily reported for \pm 2.469 ) because the observed correlation was negative $(r=-0.40)$ whereas the one-tailed t-test could only detect positive correlations (Cohen, 2013). It is unlikely this outcome reflects low statistical power because the correlation was in the opposite direction from what was predicted, and the test would have been statistically significant had a negative correlation been predicted.

\section{Discussion}

The present experiment evaluated three predictions derived from the Embodied Simulation Hypothesis: (1) Preventing participants from generating certain kinds of information about their reach capabilities should significantly increase judgment error compared to when participants were free to generate information, (2) observers' judgments should align with observers' capabilities significantly better than with the confederate's, and (3) judgment error should significantly and positively correlate with the degree of dissimilarity between observers' and the confederate's capabilities. The present results did not support any of those predictions.

It is unlikely that our failure to support the predictions was due to low statistical power. Observed effects were in the opposite directions from what was predicted, and two of the three tests would have been statistically significant had we evaluated effects in the opposite direction from what was predicted.

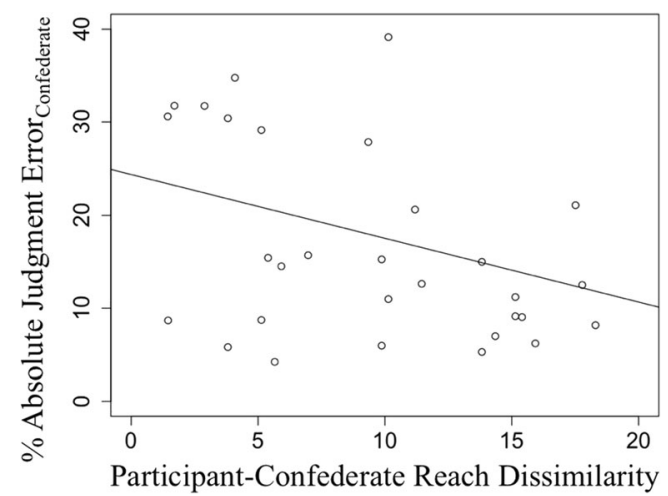

Fig. 3 Relationship between Absolute Judgment Error ${ }_{\text {Confederate }}$ and Participant-Confederate Reach Dissimilarity 


\section{Contributions}

The lack of statistically significant effects prevents definitive claims regarding our predictions. Nevertheless, the present research makes several important contributions.

First, it introduces a novel means for evaluating the Embodied Simulation Hypothesis, provides initial tests of related predictions, and corroborates Fischer (2003), who tested a variant of our third prediction. Based on the Embodied Simulation Hypothesis, Fischer predicted a positive correlation between absolute judgment error and observers' height (Experiments 1 and 2) and arm length (Experiment 3) when observers judged a short actor, and a negative correlation between those variables when observers judged a tall actor. Fischer failed to find consistent evidence for the predicted correlations, which is consistent with the present results.

Second, it motivates questions about embodied simulation. For example, what role does information pick-up play in embodied simulation? The predictions tested in the present experiment are logically consistent with standard accounts of the Embodied Simulation Hypothesis (Gallese \& Sinigaglia, 2011). However, they presume information pick-up plays a role in embodied simulation. To our knowledge, advocates of the Embodied Simulation Hypothesis have not explained how information pick-up relates to embodied simulation. Thus, the present research motivates refinement of that hypothesis. Further, what factors influence whether people perceive others' affordances via embodied simulation? Creem-Regehr, Gagnon, Geuss, and Stefanucci (2013) argued observers base judgments about an actor's affordance on the actor's affordance when there is sufficient information about the actor's affordance and on the observer's affordance (embodied simulation) when there is insufficient information about the actor's affordance, which might explain why certain studies find support for embodied simulation (Ramenzoni et al., 2008b) and others (the present experiment) fail to support embodied simulation. Addressing that issue will require an understanding of what information is necessary and sufficient to perceive an actor's affordances. Relevant information may vary between body-scaled and action-scaled affordances, which are largely determined by relations between the actor's body dimensions or kinematics and the environment, respectively (Fajen, Riley, \& Turvey, 2009).

Third, it motivates questions about whether people pick up the same information when perceiving their own or others' affordances. Jones and Widlus (in press) found observers made more erroneous judgments about whether they could reach something when their arms were restricted compared to when they were not. Mark (2007) argued observers can perceive actors' affordances because observers pick up the same information actors pick up when actors perceive their own affordances. If so, then observers should make more erroneous judgments about whether an actor could reach something when observers' arms were restricted compared to when they are not. However, the present results failed to support that possibility. That suggests how one picks up information about one's own affordances might differ from how one picks up information about others' affordances. In other words, it suggests observers might not perceive actors' affordances by picking up the same information actors pick up when actors perceive their own affordances.

\section{Future research}

A useful follow-up experiment would fully cross judgment type (observers judge own affordances vs. observers judge actors' affordances), arm restriction (observers' arms unrestricted vs. restricted), and actor information (information about actors' affordances present vs. absent). That would afford more definitive tests of our predictions because replicating the contrasting results reported in Jones and Widlus (in press) and the present experiment would result in a significant interaction between judgment type and arm restriction. In addition, it would evaluate whether that interaction depends on the available information about the actor (Creem-Regehr et al., 2013).

\section{Open practices statement}

This experiment was not preregistered. Data from the experiment reported here can be downloaded from the Texas Tech University Dataverse: doi:10.18738/T8/EY3NFE.

Author notes This research was supported in part by the US Air Force Office of Scientific Research (AFOSR) under AOARD Project\# 161OA071. Opinions, findings, and conclusions are those of the authors and do not necessarily reflect the views of the US AFOSR.

\section{References}

Cohen, J. (2013). Statistical power analysis for the behavioral sciences. Routledge.

Creem-Regehr, S. H., Gagnon, K. T., Geuss, M. N., \& Stefanucci, J. K. (2013). Relating spatial perspective taking to the perception of other's affordances: Providing a foundation for predicting the future behavior of others. Frontiers in Human Neuroscience, 7, 596.

Fajen, B. R., Riley, M. A., \& Turvey, M. T. (2009). Information, affordances, and the control of action in sport. International Journal of Sport Psychology, 40(1), 79.

Fischer, M. H. (2003). Can we correctly perceive the reaching range of others? British Journal of Psychology, 94(4), 487-500.

Gallese, V., \& Sinigaglia, C. (2011). What is so special about embodied simulation? Trends in Cognitive Sciences, 15(11), 512-519.

Gibson, J. J. (1979/1986). The ecological approach to visual perception. Hillsdale: Lawrence Erlbaum Associates.

Heft, H. (1993). A methodological note on overestimates of reaching distance: Distinguishing between perceptual and analytical judgments. Ecological Psychology, 5(3), 255-271. 
Jones, K. S., \& Widlus, B. P. (in press). Do exploratory arm movements contribute to reach-ability judgments? Quarterly Journal of Experimental Psychology.

Kirk, R. E. (1995). Experimental design: Procedures for the behavioral sciences (3rd ed.). Pacific Grove: Brooks/Cole.

Mantel, B., Stoffregen, T. A., Campbell, A., \& Bardy, B. G. (2015). Exploratory movement generates higher-order information that is sufficient for accurate perception of scaled egocentric distance. PLoS One, 10(4), e0120025.

Mark, L. S. (2007). Perceiving the actions of other people. Ecological Psychology, 19(2), 107-136.

Mark, L. S., Balliett, J. A., Craver, K. D., Douglas, S. D., \& Fox, T. (1990). What an actor must do in order to perceive the affordance for sitting. Ecological Psychology, 2(4), 325-366.

Ramenzoni, V., Riley, M. A., Davis, T., Shockley, K., \& Armstrong, R. (2008a). Tuning in to another person's action capabilities: Perceiving maximal jumping-reach height from walking kinematics. Journal of Experimental Psychology: Human Perception and Performance, 34(4), 919-928.

Ramenzoni, V. C., Riley, M. A., Shockley, K., \& Davis, T. (2008b). Carrying the height of the world on your ankles: Encumbering observers reduces estimates of how high an actor can jump. The Quarterly Journal of Experimental Psychology, 61(10), 1487-1495.

Ramenzoni, V. C., Riley, M. A., Shockley, K., \& Davis, T. (2008c). An information-based approach to action understanding. Cognition, 106(2), 1059-1070.

Stevens J. P. (2009). Applied Multivariate Statistics for the Social Sciences. Mahwah: Lawrence Erlbaum Associates, Publishers.

Stoffregen, T. A. (2003). Affordances as properties of the animalenvironment system. Ecological Psychology, 15(2), 115-134.

Stoffregen, T. A., Gorday, K. M., Sheng, Y. Y., \& Flynn, S. B. (1999). Perceiving affordances for another person's actions. Journal of Experimental Psychology: Human Perception and Performance, 25(1), 120-136.

Turvey, M. T., \& Carello, C. (2011). Obtaining information by dynamic (effortful) touching. Philosophical Transactions of the Royal Society B: Biological Sciences, 366(1581), 3123-3132.

Publisher's note Springer Nature remains neutral with regard to jurisdictional claims in published maps and institutional affiliations. 